\title{
Incidence of Neonatal Brachial Plexus Palsy in Australia 1993 - 2013: a population-based study
}

\author{
Peter Scott ${ }^{1,2}$ and Stephen J Robson ${ }^{1,2 *}$ \\ ${ }^{1}$ Australian National University Medical School, Garran, ACT, 2605, Australia \\ ${ }^{2}$ Centenary Hospital for Women and Children, Garran, ACT, 2605, Australia
}

\begin{abstract}
Background: Neonatal brachial plexus palsy (NBPP) is a rare but important adverse outcome of birth and its management is expensive and time consuming, with a permanent disability resulting in approximately $10 \%$ of cases. There is evidence from pooled data that the rate of NBPP may be falling. We used national data to determine whether this is a true finding.
\end{abstract}

Methods: Data from mandated Australian national data collections during the period 1993 until 2013 were searched for injury to the brachial plexus, Erb's paralysis due to birth trauma, and other brachial plexus birth trauma. As a denominator, the total number of live births was obtained for each relevant year. To quantify the risk factors we obtained the number of caesarean sections, instrumental deliveries, live born babies with birth weights of 4000 grams or more, and the proportion of pregnancies complicated by diabetes. Linear regressions and Pearson product-moment correlation coefficients were calculated.

Results: The incidence rate of NBPP fell significantly over the 20 year study period. There was no change in the rate of macrosomic babies at birth. The rate of caesarean birth and the percentage of instrumental vaginal deliveries of live born babies increased and there was a significant increase in the percentage of pregnancies complicated by. The strongest correlation was with the increased rate of caesarean section.

Conclusion: This study has revealed a significant reduction in the incidence of NBPP nationally. It seems likely that the steady increase in the rate of caesarean section in Australia has been associated with the decline in the rate of NBPP at a national level.

\section{Background}

Although rare, neonatal brachial plexus palsy (NBPP) is an important adverse outcome of birth. Also known as Erb's palsy, NBPP is characterised by a traction injury to the $\mathrm{C} 5$ and $\mathrm{C} 6$ nerve roots that results in an adducted and internally rotated shoulder with the elbow in extension and the forearm in pronation - colloquially known as the "waiter's tip" position [1]. Although NBPP resolves partially or completely within a year of birth in $90 \%$ of cases [2] the management required may include investigations such as electrodiagnostic imaging, nerve conduction studies and imaging, and paediatricians will be involved in prescribing treatments such intensive physical therapy and sometimes neurosurgery. For these reasons the management of NBPP is expensive, time consuming, and a source of great stress not only to parents but also to obstetricians [3]. The incidence rate of NBPP in developed countries has been estimated at between 1.3 and 1.5 per 1000 births, with a rate at one year estimated at between 1.1 and 3.7 per 10000 births [1]. A study using estimates of the overall rate of NBPP in publications from different times has suggested that the rate of NBPP may be falling. We set out to use national data from Australia to determine whether this is a true finding, and whether such a change was associated with underlying changes in birth in Australia.

\section{Methods}

Data were obtained from the mandated national data collections of the Australian Institute of Health and Welfare (AIHW) for the period 1993 until 2013. The AIHW national dataset is collected under the auspice of the Australian Health Ministers' Advisory Council (AHMAC) through the National Health Information Agreement. These data are collected as specified in the National Minimum Data Sets relating to hospitals and day procedure facilities, pooling data supplied by state and territory health authorities. The database for hospital separations was classified by the ICD-9-AM coding system for the period 1993 to 1998. During this epoch, we searched for and extracted data relating to code 767.6 (birth trauma - injury to the brachial plexus). From 1998, classification was changed to according to the ICD-10-AM, in which we searched for and extracted diagnoses of either P14.0 (Erb's paralysis due to birth trauma) or P14.6 (other brachial plexus birth trauma). As a denominator, the total number of live births was obtained from the AIHW Australia's Mothers and Babies dataset (published annually as the Australia's Mothers and Babies report) for each relevant year.

To quantify the known risk factors for NBPP we obtained the total number of caesarean sections, the number of instrumental vaginal deliveries, and the number of live born babies with a birthweight of either 4000-4499 grams, or 4500 grams or more in Australia. Unfortunately data regarding the proportion of pregnancies complicated by diabetes is incomplete at a national level, so we used the dataset from the state of New South Wales (which is the largest single jurisdiction and, over the study period, had approximately one third of all births in Australia

Correspondence to: Stephen Robson, Centenary Hospital for Women and Children, PO Box 11, Woden, ACT, 2606, Australia, Tel: +61 26282 3033; Fax: +61 26281 2899; E-mail: stephen.robson@anu.edu.au

Received: January 09, 2016; Accepted: February 05, 2016; Published: February 08,2016 
from a peak of $34.0 \%$ in $1999-2000$ to a low of $31.2 \%$ in $2012-2013$ ). National data regarding maternal obesity were only available for the latter three years of the study so could not be included. The rate of induction of labour for diabetic pregnancies was not recorded over the whole duration of the study, and the only data available were for the jurisdiction of New South Wales for the period 2007 to 2013 inclusive.

The extracted data were entered into Excel $^{\mathrm{pm}}$ spreadsheets. Linear regressions were undertaken with calculation of the correlation coefficient $\mathrm{R}$, adjusted $\mathrm{R}^{2}\left(\mathrm{aR}^{2}\right)$, and $p$-values. To test for linear correlation between the rate of NBPP and the candidate variables we calculated the Pearson product-moment correlation coefficient (Pearson's $r$ ). This study received approval from the Australian National University Human Research Ethics Committee (protocol 2015/347).

\section{Results}

The incidence rate of NBPP at neonatal discharge (cases per 1000 live births) fell significantly over the 20 year study period, with a peak of 3.78/10000 during 1998/99 and a nadir of 1.60/10000 during 2010/11 (Figure 1). With respect to risk factors for NBPP, over the study period the percentage of live born babies with a birthweight of 4000 to 4499 grams (about 10\%) and 4500 grams or greater (about 1.8\%) did not change significantly (Figure 2). The rate of caesarean birth for live born babies increased from $19 \%$ to $32.1 \%(p<0.005)$ (Figure 3 ), and the percentage of instrumental vaginal deliveries of live born babies increased from $15 \%$ to $18.3 \%(p<0.005)$ (Figure 4$)$. Data from

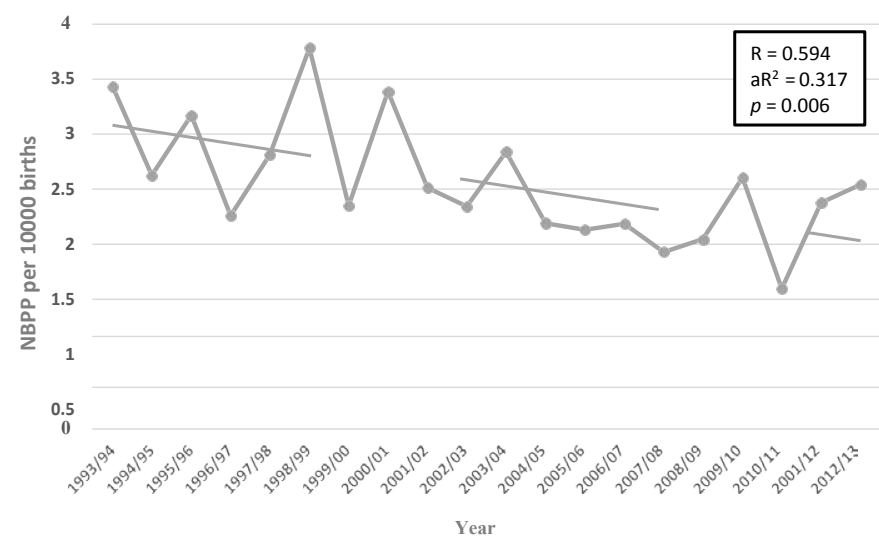

Figure 1. Incidence rate of Neonatal brachial plexus palsy (cases per 10000 livebirths) in Australia during the period 1993 to 2013.

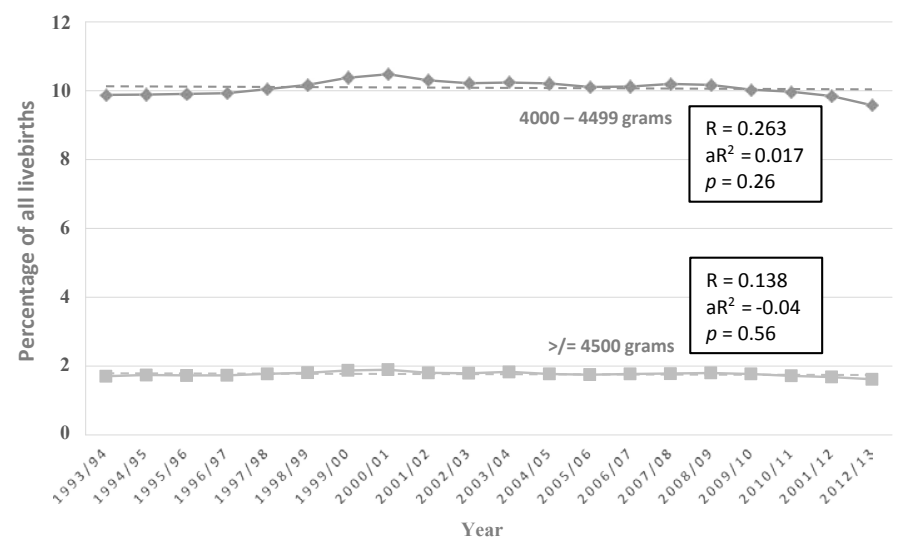

Figure 2. Percentage of live born babies with a birthweight of 4000 to 4499 grams (v) and birthweight of 4500 grams or greater ( $v$ ) in Australia during the period 1993 to2013.

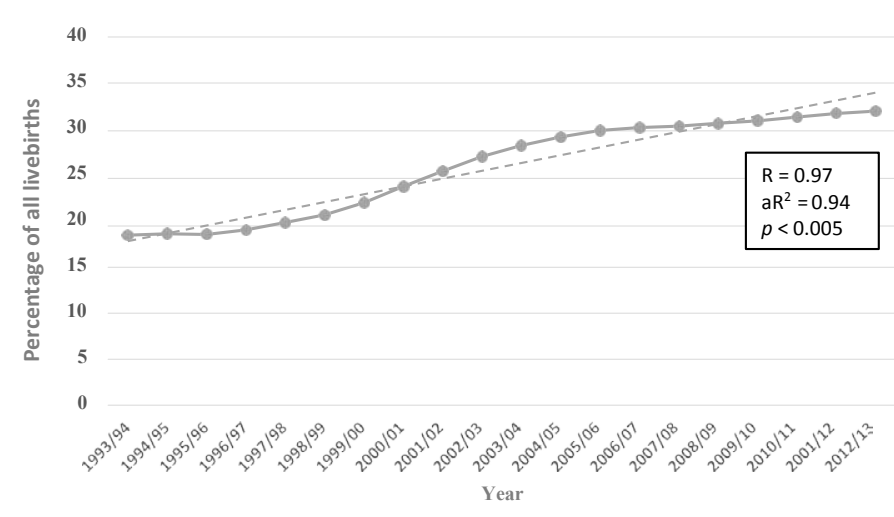

Figure 3. The percentage of live born babies delivered by caesarean section in Australia during the period 1993 to 2013 .

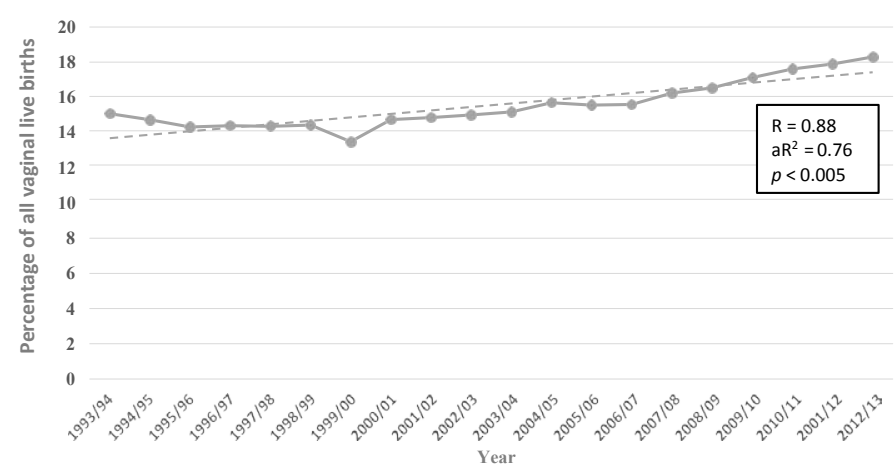

Figure 4. The percentage of all vaginal births of live born babies delivered instrumentally (ventouse + forceps) in Australia during the period 1993 to2013.

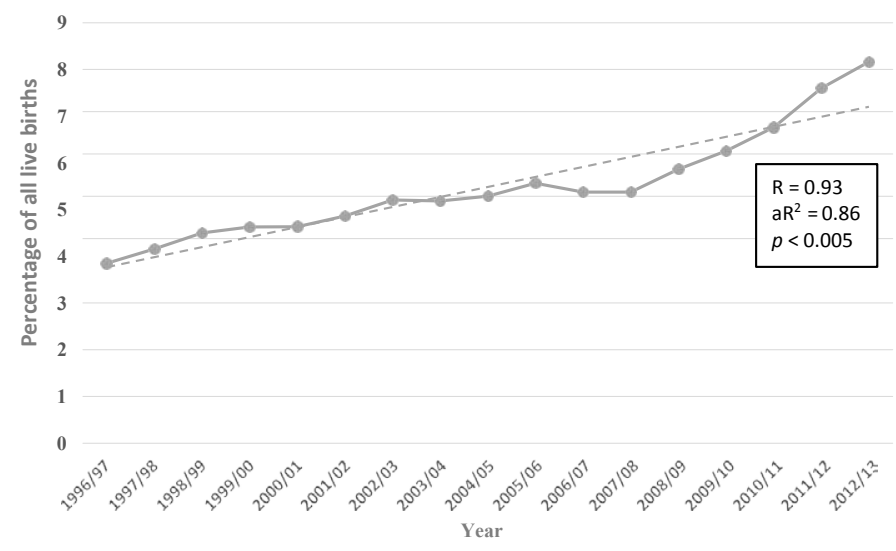

Figure 5. The percentage of all pregnancies complicated by diabetes (pre-existing diabetes + gestational diabetes) in New South Wales during the period 1996 to 2013.

New South Wales revealed a significant increase in the percentage of pregnancies complicated by diabetes (pre-existing + gestational) during the period 1996-2013 (Figure 5). There was no significant rise in the rate of induction of labour for pregnancies complicated by diabetes for the period during which data were available, 2007 to 2013 (Figure 6). A low correlation was found between the incidence rate of NBPP and the proportion of birthweights of 4000 to 4499 grams (Pearson's $r=0.52$ ) and 4500 grams or greater (Pearson's $r=0.18$ ). There was a low negative correlation with the increasing instrumental delivery rate (Pearson's $r=-0.44$ ) and diagnosis of diabetes in pregnancy in New South Wales (Pearson's $r=-0.33$ ). The strongest correlation was with 


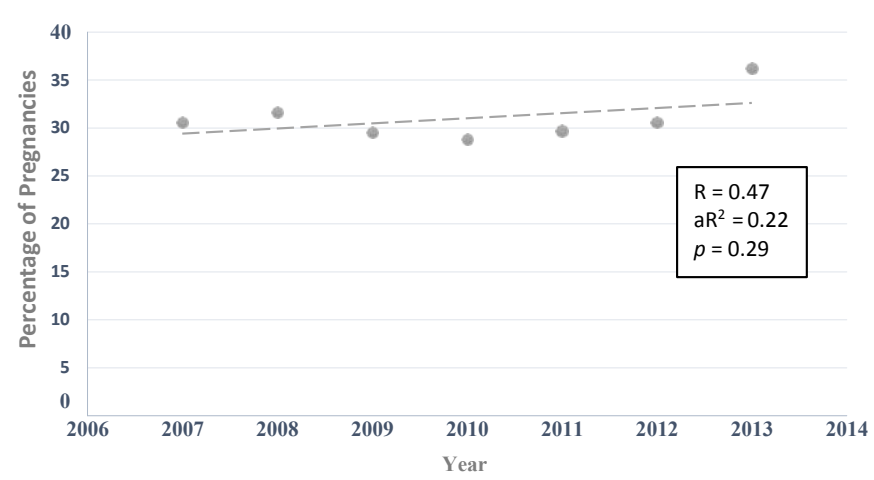

Figure 6. The percentage of all pregnancies complicated by diabetes (pre-existing diabetes + gestational diabetes) where labour was induced in New South Wales during the period 2007 to 2013.

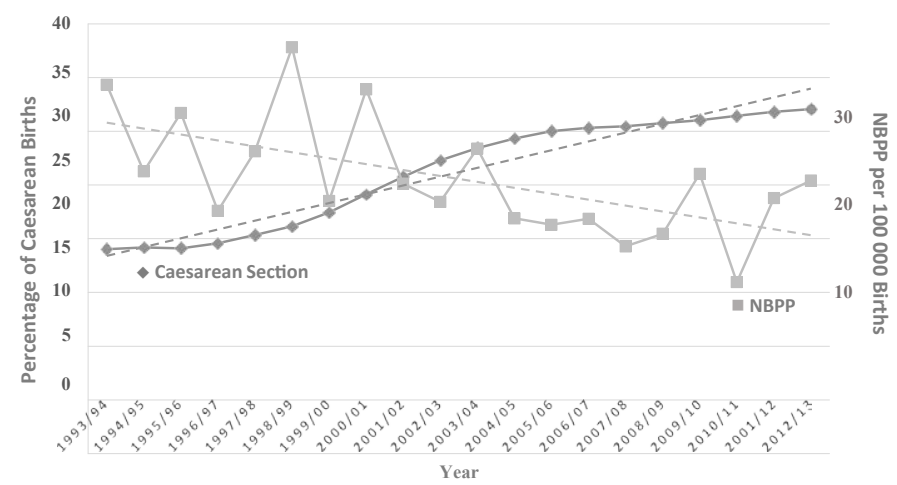

Figure 7. Overlay of the rates of caesarean section (percentage of all livebirths, scale on left) and NBPP cases (cases per 100000 births, scale on right) in Australia during the period 1993 to 2013.

the increased rate of caesarean section (Pearson's $r=-0.622$ ) (Figure 7).

\section{Discussion}

There has been a significant fall in the rate of NBPP in Australia over the 20 years until 2013 despite increases in the incidence rate of pregnancy-related risk factors of diabetes and instrumental delivery. There has been no significant change in the proportion of babies with birthweight of $4 \mathrm{Kg}$ or greater. Although data were limited to the last seven years of this study, there was no evidence that induction of labour in diabetic pregnancy was becoming more common. The strongest correlation we found was with the increasing rate of caesarean section: the increasing rate of caesarean section in Australia over the last two decades has been associated with a fall in rate of NBPP despite increases in the rate of risk factors.

NBPP has been described as 'uncommon, unpredictable, and unpreventable' [4] and this study has found that the condition is certainly uncommon with a national incidence rate in Australia that averaged 2.17 cases per 10000 births over the last three years of the study period. A review of publications using regional and institutional data have reported estimated rates of NBPP ranging from 0.6 to 3.6 cases per 1000 term births [1]. Rates of 'permanent' NBPP, similar to those reported in our national data, in a comparable period to this study range from 2.4 to 3.7 cases per 10000 births.

Fortunately, the majority of babies sustaining a NBPP have a good prognosis with the majority recovering functional use of the arm [2]. Only about one case in ten persists beyond age one, however in cases that do occur there is a substantial implication not only for quality of life of the child's parents, but also in terms of financial costs and commitment to treatment [3]. The injury typically follows an episode of shoulder dystocia at a vaginal birth [5] and ranges in severity from mild (with rapid recovery) to complete avulsion of the nerve root with no prospect of spontaneous recovery [2]. The treatment is aimed at maximization of range of movement (ROM) in all affected joints in the baby's arm to avoid development of contractures and to ensure symmetrical and appropriate overall muscle development. In severe cases where there is little functional improvement with conservative measures, microsurgical reconstruction must be considered [6-8]. Avoidance of these outcomes in clearly the most important consideration.

Because of the well-recognised association between NBPP and shoulder dystocia, the major risk factors for its occurrence are fetal macrosomia and pregnancy complicated by diabetes [9]. An association has been noted with instrumental vaginal delivery, but this is weak at best [10]. Because of the close relationship between shoulder dystocia and NBPP it should be no surprise that caesarean delivery is protective $[11,12]$. However, because of difficulties in predicting the occurrence of shoulder dystocia and the relative rarity of NBPP, there is no evidence that a liberal recourse to caesarean section where fetal macrosomia is suspected has sufficient cost-benefit to recommend it [13]. It is also possible that improvements in management of shoulder dystocia, for example drills and emergency management workshops for midwives and obstetricians, might have improved management over the study period [14]. This is, unfortunately, impossible to study at a national level.

\section{Conclusions}

NBPP has the potential for a severe burden on a family, and is typically although not always the consequence of shoulder dystocia during vaginal delivery. Although the risk factors for NBPP - fetal macrosomia, diabetes, and instrumental delivery - are well recognised, their predictive value is low and, fortunately, the incidence of NBPP is low. This study has revealed a significant reduction in the incidence of NBPP nationally in Australia despite the rate of macrosomia remaining unchanged and increases in the rate of instrumental delivery and diabetes complicating pregnancy. It seems likely that the steady increase in the rate of caesarean section in Australia has been associated with the decline in the rate of NBPP at a national level.

\section{References}

1. Chauhan SP, Blackwell SB, Ananth CV (2014) Neonatal brachial plexus palsy: incidence, prevalence, and temporal trends. Semin Perinatol 38: 210-218. [Crossref]

2. Yang LJ (2014) Neonatal brachial plexus palsy--management and prognostic factors. Semin Perinatol 38: 222-234. [Crossref]

3. Brauer CA, Waters PM (2007) An economic analysis of the timing of microsurgical reconstruction in brachial plexus birth palsy. J Bone Joint Surg Am 89: 970-978. [Crossref]

4. Hammad IA, Chauhan SP, Gherman RB, Ouzounian JG, Hill JB, et al. (2013) Neonatal brachial plexus with vaginal birth after cesarean delivery: a case-control study. $A m \mathrm{~J}$ Obstet Gynecol 208: 229. [Crossref]

5. Chauhan SP (2014) Shoulder dystocia and neonatal brachial plexus palsy: eliminating the nightmare. Semin Perinatol 38: 183. [Crossref]

6. Sunderland S (1951) A classification of peripheral nerve injuries producing loss of function. Brain 74: 491-516. [Crossref]

7. Dodds SD, Wolfe SW (2000) Perinatal brachial plexus palsy. Curr Opin Pediatr 12: 40-47. [Crossref]

8. Gilbert A (1995) Long-term evaluation of brachial plexus surgery in obstetrical palsy. Hand Clin 11: 583-594. [Crossref] 
9. Gilbert WM, Nesbitt TS, Danielsen B (1999) Associated factors in 1611 cases of brachial plexus injury. Obstet Gynecol 93: 536-540. [Crossref]

10. Ouzounian JG, Korst LM, Miller DA, Lee RH (2013) Brachial plexus palsy and shoulder dystocia: obstetric risk factors remain elusive. Am J Perinatol 30: 303-307. [Crossref]

11. al-Qattan MM, al-Kharfy TM (1996) Obstetric brachial plexus injury in subsequent deliveries. Ann Plast Surg 37: 545-548. [Crossref]

12. Gherman RB, Chauhan S, Ouzounian JG, Lerner H, Gonik B, et al. (2006) Shoulder dystocia: the unpreventable obstetric emergency with empiric management guidelines. Am J Obstet Gynecol 195: 657-672. [Crossref]

13. Rouse DJ, Owen J, Goldenberg RL, Cliver SP (1996) The effectiveness and costs of elective cesarean delivery for fetal macrosomia diagnosed by ultrasound. JAMA 276 : 1480-1486. [Crossref]

14. Draycott TJ, Crofts JF, Ash JP, Wilson LV, Yard E, et al. (2008) Improving neonatal outcome through practical shoulder dystocia training. Obstet Gynecol 112: 14-20. [Crossref]

Copyright: (C2016 Scott P. This is an open-access article distributed under the terms of the Creative Commons Attribution License, which permits unrestricted use, distribution, and reproduction in any medium, provided the original author and source are credited. 